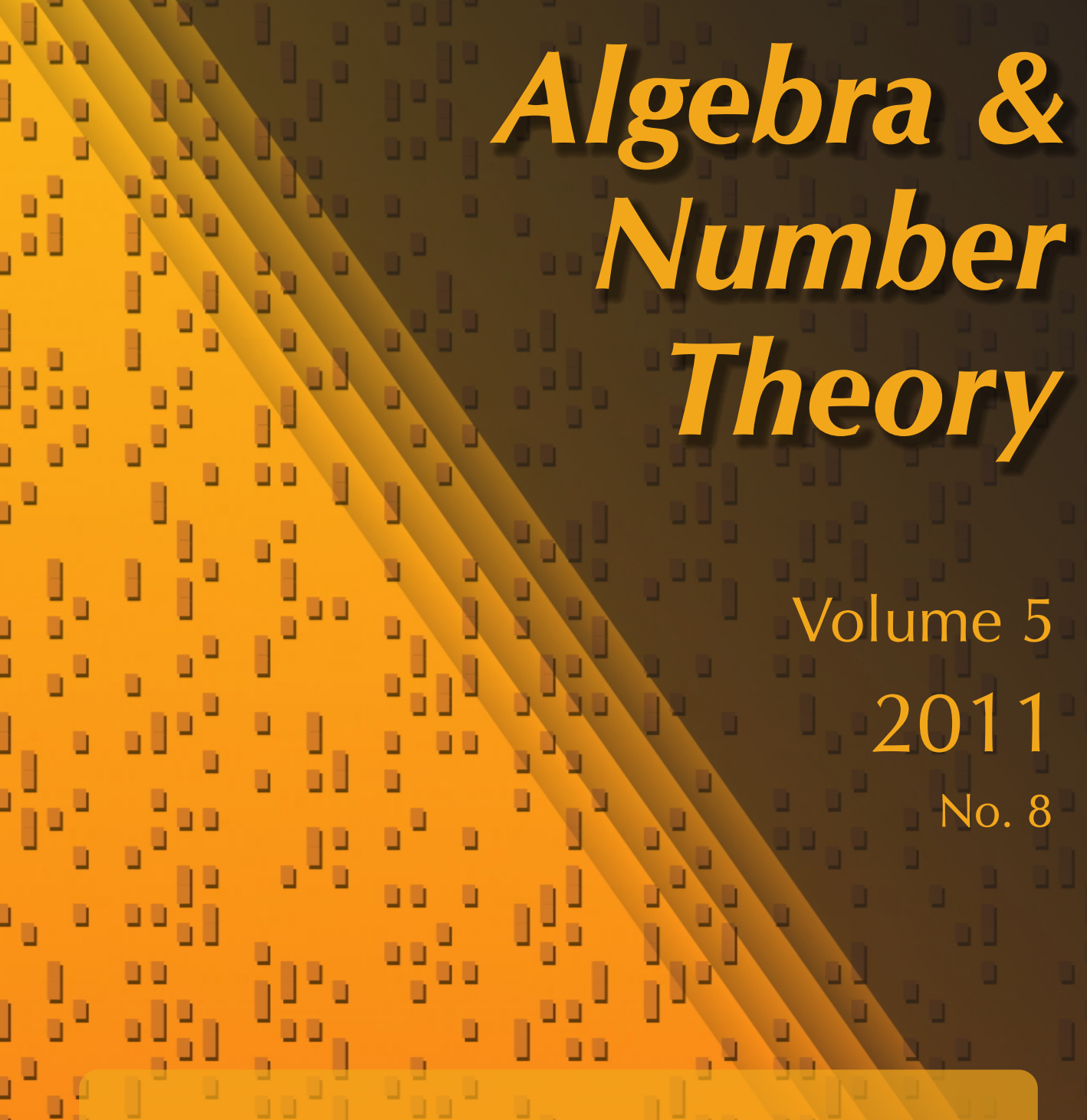

Conjecture de Shafarevitch effective pour les revêtements cycliques

\lrcorner$\lrcorner$ Robin de Jong et Gaël Rémond $\lrcorner$

」

.

\lrcorner $」 」\lrcorner\lrcorner \sqcup$ Volume 5

\lrcorner$\lrcorner\lrcorner$

\lrcorner$\lrcorner\lrcorner\lrcorner$

\lrcorner$\lrcorner\lrcorner$

\lrcorner$\lrcorner$

No. 8

J

J.

\lrcorner$\lrcorner\lrcorner$

Jathematical sciences publishers $\lrcorner$ 


\title{
Conjecture de Shafarevitch effective pour les revêtements cycliques
}

\author{
Robin de Jong et Gaël Rémond
}

\begin{abstract}
On donne une borne supérieure explicite en fonction de $K, S, g$ pour la hauteur de Faltings de la jacobienne d'une courbe $C$ de genre $g$, définie sur un corps de nombres $K$ et ayant bonne réduction en dehors d'un ensemble fini $S$ de places de $K$, pourvu que $C$ puisse s'écrire comme un revêtement cyclique de degré premier de la droite projective. La preuve repose sur le fait que les birapports des points de branchement du revêtement sont des $S$-unités, donc de hauteur bornée, et donnent un modèle plan de $C$.

We give an explicit upper bound in terms of $K, S, g$ for the Faltings height of the jacobian of a curve $C$ of genus $g$, defined over a number field $K$ and with good reduction outside a finite set $S$ of places of $K$ under the condition that $C$ can be written as a cyclic cover of prime order of the projective line. The proof rests on the fact that the cross ratios of the branch points of the cover are $S$-units, thus of bounded height, and give a plane model of $C$.
\end{abstract}

\section{Introduction}

Dans cet article, nous démontrons une version effective de la conjecture de Shafarevitch pour les courbes qui sont revêtements cycliques de degré premier de $\mathbb{P}^{1}$. Rappelons que Faltings [1983] a établi en toute généralité la version qualitative de cette conjecture (formulée comme une question dans son allocution au congrès international de Stockholm en 1962, voir [Shafarevitch 1963]).

Théorème 1.1. Soient $K$ un corps de nombres, $S$ un ensemble fini de places finies de $K$ et $g \geq 2$ un entier. Alors l'ensemble des classes d'isomorphie de courbes projectives lisses $C$ de genre $g$ sur $K$ ayant bonne réduction en dehors de $S$ est fini.

Le premier auteur est financé par une subvention VENI de l'Organisation Néerlandaise pour la Recherche Scientifique (NWO).

MSC2010: $11 \mathrm{G} 30$.

Mots-clefs : conjecture de Shafarevitch, courbe, revêtement, hauteur, réduction, birapport,

Shafarevich conjecture, curve, cover, height, reduction, cross ratio. 
La conclusion vaut également dans le cas $g=1$ lorsque l'on suppose que $C$ admet un point rationnel sur $K$, autrement dit pour les courbes elliptiques. L'énoncé est aussi vrai pour $g=0$ comme l'avait déjà observé Shafarevitch.

Une manière naturelle de quantifier cet énoncé consiste à borner la hauteur d'une telle courbe. Pour cela, plusieurs notions de hauteur peuvent être employées ; ici, pour faire un choix intrinsèque, nous utilisons la hauteur de Faltings stable de la jacobienne de notre courbe, notée $h_{\text {Falt }}(C)$.

En guise de motivation, nous mentionnons encore qu'une majoration de $h_{\text {Falt }}(C)$ dans le théorème 1.1 sans restriction sur $C$ entraînerait une version effective de la conjecture de Mordell (voir [Rémond 1999]). Toutefois la majoration explicite que nous donnons ci-dessous dans le cas très particulier des revêtements cycliques de degré premier n'a pas de conséquences directes dans cette direction (il faudrait connaitre une courbe pour laquelle la construction de Kodaira-Parshin fournit une famille de courbes qui soient toutes de tels revêtements ; mais ceci signifierait que l'on peut tracer une courbe complète dans l'espace des modules des courbes d'un genre donné qui soit entièrement contenue dans le lieu des courbes de ce type; or le dit lieu se trouve être affine, voir théorème 6.1 de [González Díez 1991], donc c'est impossible).

Pour énoncer notre résultat principal, nous devons quantifier la donnée de $K$ et $S$. Si nous mesurons classiquement $K$ par son degré $D=[K: \mathbb{Q}]$ et son discriminant absolu $\Delta=\left|\Delta_{K / \mathbb{Q}}\right|$, nous introduisons pour $S$ les quantités

$$
\Omega=\sum_{\mathfrak{p} \in S} \log N_{K / \mathbb{Q}}(\mathfrak{p})+D \log 4 \quad \text { et } \quad \Delta_{S}=\Delta e^{\Omega^{2}} .
$$

Le terme $D \log 4$ interviendra pour tenir compte des places infinies; en revanche la définition de $\Delta_{S}$ est purement ad hoc (par exemple la quantité $\Delta e^{\Omega}$ serait peut-être plus naturelle). Nous fixons une clôture algébrique $\bar{K}$ de $K$.

Théorème 1.2. Soient $K$ un corps de nombres, $S$ un ensemble fini de places finies de $K$ et $g$ un entier. Soit $C$ une courbe projective lisse de genre $g$ sur $K$ ayant bonne réduction en dehors de $S$. On suppose qu'il existe un $K$-morphisme $\pi: C \rightarrow \mathbb{P}^{1}$ dont l'extension à $\bar{K}$ est un revêtement cyclique de degré premier. Alors

$$
h_{\text {Falt }}(C) \leq 2^{2^{22} 9^{g}} \Delta_{S}^{2^{15} g^{5}} .
$$

Pour $g \geq 2$, avec les propriétés de hauteur de $h_{\text {Falt }}$, nous obtenons la finitude de l'ensemble des courbes en question, indépendamment des résultats de Faltings. Notre énoncé contient aussi le cas des courbes elliptiques et des courbes hyperelliptiques ayant un point rationnel (revêtements de degré 2 de $\mathbb{P}^{1}$ ); dans ce cas, l'énoncé de finitude était connu de Shafarevitch lui-même et de Parshin (voir [Oort 
1974] et les références). Notre démarche s'inspire de cette approche telle que formulée par Oort.

La démonstration repose sur le principe suivant. On considère les points de branchement $P_{1}, \ldots, P_{r}$ de $\pi$ sur $\bar{K}$. Ils forment une famille de points de $\mathbb{P}^{1}(\bar{K})$ stable sous l'action de $\operatorname{Gal}(\bar{K} / K)$. On leur associe ensuite leurs birapports, soit $6\left(\begin{array}{l}r \\ 4\end{array}\right)$ éléments de $\bar{K}^{\times} \backslash\{1\}$ sur lesquels agissent les involutions $x \mapsto 1-x$ et $x \mapsto x^{-1}$ ainsi que le groupe $\operatorname{Gal}(\bar{K} / K)$. Pour un tel birapport $b$, on note $L=K(b)$ et $S^{\prime}$ l'ensemble fini des places de $L$ formé des places divisant une place de $S$ et de celles divisant $p=\operatorname{deg} \pi$. On montre alors que $b$ est un $S^{\prime}$-entier et que $L / K$ n'est pas ramifiée en dehors de $S^{\prime}$. Cet argument s'inspire directement du cas des courbes hyperelliptiques traité par Oort [1974]. Il se base principalement sur le fait que les points de ramification de $\pi$ (dans $C$ ) correspondent à des points de $p$-torsion (dans Jac $C$ ) et que cette $p$-torsion s'étend en un schéma étale au-dessus de $\operatorname{Spec} 0_{S^{\prime}}$ (voir partie 2).

Comme $K(b)=K(1-b)=K\left(b^{-1}\right)$, ce qui précède fait en réalité de $(1-b, b)$ un couple de $S^{\prime}$-unités satisfaisant l'équation $x+y=1$. Cette équation aux $S$ unités a été largement étudiée et l'on sait grâce à la théorie des formes linéaires de logarithmes borner la hauteur des solutions. De manière précise, nous employons ici une majoration explicite due à Győ́ry et Yu [2006]. Elle fait apparaître le régulateur de $L$ que nous majorons à l'aide du discriminant $\Delta_{L / \mathbb{Q}}$ (résultat de Lenstra [1992]) puis nous contrôlons celui-ci en fonction de $\Delta$ et $\Omega$ en utilisant le fait que $L / K$ n'est ramifiée qu'aux places de $S^{\prime}$. Tout ceci conduit à $h(b) \leq \Delta_{S}^{(8 g)^{5}}$ (voir partie 3).

Pour la dernière étape, nous travaillons uniquement sur $\bar{K}$. Nous pouvons alors opérer un automorphisme de $\mathbb{P}^{1}$ de sorte que 0,1 et $\infty$ soient des points de branchement de $\pi$. Les $r-3$ autres se retrouvent alors être parmi les birapports que nous avons étudiés et dont nous avons borné la hauteur. Maintenant, comme le corps de fonctions de $C_{\bar{K}}$ est une extension de Kummer de $\bar{K}(X)$, nous voyons que notre courbe admet un modèle plan (singulier) d'équation affine $Y^{p}=\prod_{i=1}^{r-1}\left(X-b_{i}\right)^{a_{i}}$ où $1 \leq a_{i} \leq p-1$ et les $b_{i}$ sont les abscisses des points de branchement différents de $\infty$. Comme $h\left(b_{i}\right) \leq \Delta_{S}^{(8 g)^{5}}$, on majore immédiatement la hauteur (naïve) de cette équation. Les résultats de [Rémond 2010] permettent alors de contrôler un plongement de $C$ dans $\mathbb{P}_{\bar{K}}^{3}$ puis la hauteur thêta de Jac $C$. Finalement, une comparaison due à Bost et David (voir [Pazuki 2012]) fait le lien avec la hauteur de Faltings (voir partie 4).

\section{Bonne réduction}

Nous nous plaçons sous les hypothèses du théorème 1.2. Notons $p=\operatorname{deg} \pi$ et $\sigma: C_{\bar{K}} \rightarrow C_{\bar{K}}$ un générateur du groupe de Galois de $\pi_{\bar{K}}$. Si $Q_{1}, \ldots, Q_{r}$ sont les 
points de ramification de $\pi$ (dans $C(\bar{K})$ ), nous écrivons $P_{i}=\pi\left(Q_{i}\right)$ les points de branchement correspondants. Ils sont deux à deux distincts puisque $\pi^{-1}\left(P_{i}\right)$ est un ensemble de cardinal $<p$ sur lequel $\sigma$ (d'ordre $p$ ) agit transitivement donc un singleton. Ceci revient à dire que l'indice de ramification de chaque $Q_{i}$ vaut $p$ et la formule d'Hurwitz donne donc

$$
2 g-2=-2 p+r(p-1) \quad \Longleftrightarrow 2 g=(r-2)(p-1) .
$$

Nous excluons le cas où $g=0$ (puisque $\operatorname{Jac} C=0$ on a $h_{\text {Falt }}(C)=0$ et le théorème est trivial) donc la relation précédente fournit $3 \leq r \leq 2 g+2$ et $2 \leq p \leq 2 g+1$.

Nous utilisons quelques faits élémentaires (et classiques) sur le birapport. Le birapport de quatre éléments distincts $a, b, c, d$ d'un corps $k$ s'écrit

$$
\operatorname{Bir}(a, b, c, d)=\frac{c-a}{c-b} \cdot \frac{d-b}{d-a} .
$$

On l'étend immédiatement aux points distincts de $\mathbb{P}^{1}(k)=k \cup\{\infty\}$ (par exemple $\operatorname{Bir}(\infty, b, c, d)=(d-b) /(c-b))$. On obtient toujours un élément de $k \backslash\{0,1\}$. En particulier $\operatorname{Bir}(\infty, 0,1, x)=x$ pour tout $x \in k \backslash\{0,1\}$. On vérifie aussi facilement que le birapport est invariant par un automorphisme de $\mathbb{P}^{1}$. On a encore

$$
\operatorname{Bir}(a, b, d, c)=\operatorname{Bir}(a, b, c, d)^{-1} \quad \text { et } \quad \operatorname{Bir}(a, c, b, d)=1-\operatorname{Bir}(a, b, c, d) .
$$

Enfin

$$
\operatorname{Bir}(a, b, c, d)=\operatorname{Bir}(b, a, d, c)=\operatorname{Bir}(c, d, a, b)=\operatorname{Bir}(d, c, b, a),
$$

ce qui entraîne que les 24 birapports formés en permutant $a, b, c, d$ prennent au plus 6 valeurs.

Nous formons l'ensemble des birapports des points $P_{i}$ de $\mathbb{P}^{1}(\bar{K})$ :

$$
\mathscr{B}=\left\{\operatorname{Bir}\left(P_{i}, P_{j}, P_{k}, P_{\ell}\right) \mid 1 \leq i, j, k, \ell \leq r \text { deux à deux distincts }\right\} .
$$

Par ce qui précède, $\operatorname{Card} \mathscr{B} \leq 6\left(\begin{array}{l}r \\ 4\end{array}\right)$ et $\mathscr{B}$ est stable par les involutions $x \mapsto x^{-1}$ et $x \mapsto 1-x$. De plus, comme $\pi$ est défini sur $K$, l'ensemble $\left\{P_{1}, \ldots, P_{r}\right\}$ est stable sous l'action de $\operatorname{Gal}(\bar{K} / K)$ et il en va donc de même de $\mathscr{B}$. En particulier tout élément de $\mathscr{B}$ est de degré au plus $6\left(\begin{array}{l}r \\ 4\end{array}\right)$. Bien entendu, si $r=3$, l'ensemble $\mathscr{B}$ est vide et l'on peut passer directement à la partie 4.

Pour toute extension finie $L$ de $K$ on note $S_{L}$ l'ensemble des places de $L$ qui divisent une place de $S$ ou $p$. L'objectif de cette partie consiste à montrer l'énoncé suivant.

Proposition 2.1. Pour tout $b \in \mathscr{B}$, l'extension $K(b) / K$ est non ramifiée en dehors de $S_{K(b)}$ et b est un $S_{K(b)}$-entier.

Bien entendu, pour établir ceci, nous pouvons nous contenter d'exhiber une extension $K^{\prime}$ de $K$ non ramifiée en dehors de $S_{K^{\prime}}$, contenant $b$ comme $S_{K^{\prime}}$-entier. 
C'est ce que nous faisons en choisissant pour $K^{\prime}$ la plus petite extension de $K$ sur laquelle tous les points $Q_{i}$ sont rationnels. Vu la définition, nous avons bien $\mathscr{B} \subset K^{\prime}$. Fixons ensuite une place finie $v^{\prime} \notin S_{K^{\prime}}$ de $K^{\prime}$; notons $\mathscr{O}_{v^{\prime}}$ son anneau de valuation, $v=\left.v^{\prime}\right|_{K}$ et $\mathrm{O}_{v}=\mathscr{O}_{v^{\prime}} \cap K$. Pour conclure, il nous suffit de montrer sous ces hypothèses que $\mathscr{O}_{v^{\prime}} / \mathcal{O}_{v}$ n'est pas ramifiée et $\mathscr{B} \subset \mathscr{O}_{v^{\prime}}$. Ces deux propriétés vont résulter de l'étude de différents modèles sur $O_{v}$ et $O_{v^{\prime}}$ que nous introduisons maintenant.

Tout d'abord, puisque $v \notin S$, la courbe $C \rightarrow \operatorname{Spec} K$ s'étend en un morphisme

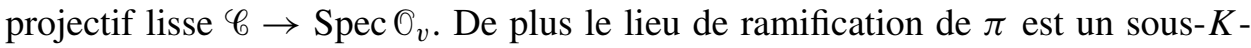
schéma $Y$ de $C$ et nous considérons son adhérence $\mathscr{Y} \subset \mathscr{C}$ (sous-schémas fermés réduits). Nous notons ensuite $C^{\prime}, Y^{\prime}, \mathscr{C}^{\prime}, \mathscr{Y}^{\prime}$ l'extension de ces objets à $K^{\prime}$ ou $\mathfrak{O}_{v^{\prime}}$. Par définition de $K^{\prime}$, le schéma $Y^{\prime} \simeq \operatorname{Spec}\left(K^{\prime}\right)^{r}$ est l'union des points rationnels $Q_{1}, \ldots, Q_{r}$. Écrivons encore $J^{\prime}$ la jacobienne de $C^{\prime}$ et $\mathscr{g}^{\prime}$ son modèle de Néron sur $\mathcal{O}_{v^{\prime}}$. Nous plongeons $C^{\prime}$ dans $J^{\prime}$ à l'aide du point rationnel $Q_{1}$ (application $\left.Q \mapsto(Q)-\left(Q_{1}\right)\right)$ et ce morphisme $C^{\prime} \rightarrow J^{\prime}$ s'étend de manière unique en $\mathscr{C}^{\prime} \rightarrow \mathscr{g}^{\prime}$ par la propriété de Néron.

Nous pouvons alors énoncer le lemme-clef de cette partie (comparer avec les arguments de Oort [1974], lemmes 2.1 et 2.2, dans le cas hyperelliptique).

Lemme 2.1. Le morphisme $\mathrm{y}^{\prime} \rightarrow \mathrm{Spec}_{\mathrm{O}_{v^{\prime}}}$ est étale.

Démonstration. Nous avons $\pi^{*}\left(P_{i}\right)=p\left(Q_{i}\right)$ en termes de diviseurs sur $C^{\prime}$ donc $p\left(Q_{i}\right) \equiv p\left(Q_{j}\right)$ pour $1 \leq i, j \leq r$. Par suite $p\left(\left(Q_{i}\right)-\left(Q_{1}\right)\right)=0$ dans $J^{\prime}$ pour $1 \leq i \leq r$ et ceci signifie exactement que $Y^{\prime} \rightarrow C^{\prime} \rightarrow J^{\prime}$ se factorise à travers le sousschéma $J_{p}^{\prime}$, noyau de la multiplication par $p$ dans $J^{\prime}$. Comme $Y^{\prime}$ et $J_{p}^{\prime}$ sont discrets et réduits, l'immersion $a: Y^{\prime} \rightarrow J_{p}^{\prime}$ est à la fois ouverte et fermée. Maintenant l'hypothèse $v^{\prime} \notin S_{K^{\prime}}$ assure que le corps résiduel de $0_{v^{\prime}}$ n'est pas de caractéristique $p$ donc $\mathscr{F}_{p}^{\prime}$ est étale sur $\mathscr{O}_{v^{\prime}}$. En particulier, chaque composante connexe de $\mathscr{F}_{p}^{\prime}$ est intègre et coïncide donc avec l'adhérence (dans $\mathscr{J}^{\prime}$ ) d'un point de $J_{p}^{\prime}$. Ainsi $a$ s'étend en une immersion ouverte et fermée $\mathscr{Y}^{\prime} \rightarrow \mathscr{F}_{p}^{\prime}$ (si $\mathscr{L}^{\prime}$ est une composante connexe de $\mathscr{F}_{p}^{\prime}$, les seuls sous-schémas fermés de $\mathscr{L}^{\prime}$ qui induisent un sous-schéma ouvert sur la fibre générique sont $\varnothing$ et $\left.\mathscr{E}^{\prime}\right)$. Une immersion ouverte étant étale, il en va de même de Yy'. $^{\prime}$.

Nous pouvons d'ores et déjà déduire de ce lemme la propriété de non-ramification. En effet, il entraîne que Yy est étale sur $\operatorname{Spec} \mathcal{O}_{v}$ et ceci signifie exactement que, dans chacun des corps résiduels des points de $Y$, la place $v$ ne se ramifie pas. Or, par définition, $K^{\prime}$ est le compositum de ces corps donc $\mathbb{O}_{v^{\prime}} / \mathcal{O}_{v}$ est effectivement non ramifiée.

Soit maintenant $K^{\prime \prime}$ une extension finie de $K^{\prime}$ sur laquelle l'automorphisme $\sigma$ fixé plus haut est défini. Soit $v^{\prime \prime}$ une place de $K^{\prime \prime}$ au-dessus de $v^{\prime}$. Nous notons $C^{\prime \prime}$, $J^{\prime \prime}, \mathscr{C}^{\prime \prime}, \mathscr{Y}^{\prime \prime}$ et $\mathscr{g}^{\prime \prime}$ les extensions de $C^{\prime}, J^{\prime}, \mathscr{C}^{\prime}, \mathscr{Y}^{\prime}$ et $\mathscr{g}^{\prime}$ à $K^{\prime \prime}$ ou $O_{v^{\prime \prime}}$. Nous notons 
par la même lettre l'automorphisme $\sigma: C^{\prime \prime} \rightarrow C^{\prime \prime}$ et son extension en un automorphisme $J^{\prime \prime}$ puis de $\mathscr{F}^{\prime \prime}$ (par propriété de Néron) et enfin de $\mathscr{C}^{\prime \prime}$ (par restriction à l'adhérence de $C^{\prime \prime}$ dans $\left.\mathscr{F}^{\prime \prime}\right)$. Bien entendu, à chaque étape, $\sigma$ vérifie $\sigma^{p}=\mathrm{id}$. Notons $G$ le groupe d'automorphismes de $\mathscr{C}^{\prime \prime}$ engendré par $\sigma$.

\section{Lemme 2.2. Le quotient $\mathscr{G}^{\prime \prime} / G$ existe et est isomorphe à $\mathbb{P}_{\mathscr{O}^{\prime \prime}}^{1}$.}

Démonstration. Pour l'existence, d'après le théorème 4.12 de [Lønsted et Kleiman 1979], il suffit de vérifier que $G$ agit fidèlement sur la fibre spéciale de $\mathscr{C}^{\prime \prime}$. Si ce n'était pas le cas, $\sigma$ induirait l'identité sur cette fibre spéciale $\mathscr{C}_{s}^{\prime \prime}$ donc également sur celle de $\mathscr{F}^{\prime \prime}$ notée $\mathscr{F}_{s}^{\prime \prime}$. Ceci est absurde car pour un schéma abélien l'application de restriction End $\mathscr{F}^{\prime \prime} \rightarrow$ End $\mathscr{F}_{s}^{\prime \prime}$ est toujours injective (voir [Lang 1983] page 45). Notons donc $\mathscr{P}=\mathscr{C}^{\prime \prime} / G$ ce quotient et $\mathscr{P}_{s}$ sa fibre spéciale qui est une courbe lisse de genre $g^{\prime}$. Le revêtement $\mathscr{C}_{s}^{\prime \prime} \rightarrow \mathscr{P}_{s}$ de groupe $G$ a au moins $r$ points de ramification : ceux de la fibre spéciale du schéma $y^{\prime \prime}$. Par suite la formule d'Hurwitz (il n'y a pas de ramification sauvage) donne

$$
2 g-2=p\left(2 g^{\prime}-2\right)+r^{\prime}(p-1) \quad \Longleftrightarrow 2 p g^{\prime}+\left(r^{\prime}-r\right)(p-1)=0
$$

où $r^{\prime} \geq r$ est le nombre de points de ramification. On conclut $r^{\prime}=r$ et $g^{\prime}=0$. Ainsi $\mathscr{P}$ est une famille de courbes de genre 0 et elle admet une section : il suffit de considérer un point de branchement (en d'autres termes la composée d'une section de $\mathscr{Y}^{\prime \prime}$ avec $\mathscr{Y}^{\prime \prime} \rightarrow \mathscr{C}^{\prime \prime} \rightarrow \mathscr{P}$ ). La proposition 3.3 de [Lønsted et Kleiman 1979] assure alors que $\mathscr{P} \simeq \mathbb{P}(\mathscr{E})$ pour un faisceau localement libre $\mathscr{E}$ de rang 2 sur Spec $\mathcal{O}_{v^{\prime \prime}}$. Par principalité de $\mathcal{O}_{v^{\prime \prime}}$, ce faisceau $\mathscr{E}$ est libre donc $\mathscr{P} \simeq \mathbb{P}_{\mathscr{O}_{v^{\prime \prime}}}^{1}$.

Nous déduisons facilement $\mathscr{B} \subset \mathrm{O}_{v^{\prime \prime}}$ de cet énoncé. Il fournit en effet un morphisme $\mathscr{C}^{\prime \prime} \rightarrow \mathbb{P}_{\mathscr{O}_{v^{\prime \prime}}}^{1}$ dont la fibre générique coïncide avec l'extension de $\pi$ à $K^{\prime \prime}$ modulo un automorphisme de $\mathbb{P}_{K^{\prime \prime}}^{1}$. Comme un tel automorphisme ne modifie pas l'ensemble $\mathscr{B}$, nous pouvons considérer que $P_{1}, \ldots, P_{r}$ sont les points de branchement de ce morphisme. Quitte à faire un automorphisme de $\mathbb{P}_{\mathscr{O}_{v^{\prime \prime}}}^{1}$ nous supposons même $P_{1}=\infty$. Comme les points de branchement sont toujours distincts dans la fibre spéciale (cela vient encore de ce que $\mathrm{y}^{\prime \prime}$ est étale et fixé par $\sigma$ ), nous en déduisons que $P_{i}=\left(e_{i}: 1\right)$ où $e_{i} \in \mathcal{O}_{v^{\prime \prime}}, i \geq 2\left(\operatorname{car} P_{1}=\infty=(1: 0)\right)$ puis $e_{i}-e_{j} \in \mathbb{O}_{v^{\prime \prime}}^{\times}$ si $2 \leq i<j$. Alors chaque élément de $\mathscr{B}$ s'écrit

$$
\frac{e_{i}-e_{k}}{e_{i}-e_{j}} \quad \text { ou } \quad \frac{e_{i}-e_{k}}{e_{i}-e_{j}} \cdot \frac{e_{\ell}-e_{j}}{e_{\ell}-e_{k}}
$$

(selon que $P_{1}$ apparaît ou non) avec $i \neq j$ et $k \neq \ell$. Ceci entraîne clairement $\mathscr{B} \subset \mathcal{O}_{v^{\prime \prime}}$ puis $\mathscr{B} \subset \mathfrak{O}_{v^{\prime \prime}} \cap K^{\prime}=\mathscr{O}_{v^{\prime}}$ et termine donc la démonstration de la proposition 2.1. 


\section{3. $S$-unités, régulateurs et discriminants}

L'objectif de cette partie est d'établir la majoration suivante de la hauteur des éléments de $\mathscr{B}$.

Proposition 3.1. Pour tout $b \in \mathscr{P}$, on $a h(b) \leq \Delta_{S}^{(8 g)^{5}}$.

Nous fixons donc un élément $b$ de $\mathscr{B}$ et notons $L=K(b)$ ainsi que $S^{\prime}=S_{L}$. Nous désignons par $d, R$ et $h$ le degré de $L$ (sur $\mathbb{Q}$ ), son régulateur et son nombre de classes. En vue d'appliquer le résultat de Győry et Yu [2006] nous écrivons encore $s$ pour le cardinal de $S^{\prime} \cup\{$ places infinies $\}, R_{S^{\prime}}$ pour le $S^{\prime}$-régulateur de $L$ et $P$ pour le maximum des $N_{L / \mathbb{Q}}(\mathfrak{p}), \mathfrak{p} \in S^{\prime}$. Nous abrégeons aussi $\log ^{\star} x=\max (1, \log x)$ pour $x>0$.

Lemme 3.1. La hauteur de b est au plus

$$
2^{15}(16 s d)^{2 s+4} P R_{S^{\prime}}\left(1+\log ^{\star} R_{S^{\prime}} / \log ^{\star} P\right) .
$$

Démonstration. Nous appliquons la proposition 2.1 aux quatre éléments $b, b^{-1}$, $1-b$ et $(1-b)^{-1}$ de $\mathscr{B}$. Ce sont donc des $S^{\prime}$-entiers et donc des $S^{\prime}$-unités. Le couple $(b, 1-b)$ appartient à $\left\{(x, y) \in\left(\mathcal{O}_{S^{\prime}}^{\times}\right)^{2} \mid x+y=1\right\}$ donc nous pouvons lui appliquer le théorème principal de [Győry et Yu 2006] avec $\alpha=\beta=1$ et $H=4$. Nous obtenons alors la borne de l'énoncé en majorant $\log (2 s) \leq \sqrt{2 s}$ et $\log ^{\star}(2 d) \leq \sqrt{2 d}$ puis $7 s+29 \leq 8 s+27$ dans l'exposant de 2 .

Nous estimons maintenant les quantités apparaissant dans cette formule. Notons $E$ un majorant de $[L: K]$. Nous choisissons $E \geq 2$ pour simplifier les calculs (in fine nous majorerons $E$ par $6\left(\begin{array}{l}r \\ 4\end{array}\right)$ ). On pose $u=E \Omega / \log 2$.

Nous avons facilement $d \leq E D \leq u$ et

$$
s \leq 2 E D+\sum_{\mathfrak{p} \in S} E \leq 2 E D+\sum_{\mathfrak{p} \in S} E \frac{\log N_{K / \mathbb{Q}}(\mathfrak{p})}{\log 2}=u
$$

car il y a au plus $E D$ places au-dessus de $p, E D$ au-dessus de $\infty$ et $E$ au-dessus de chaque place de $S$. De manière analogue, $P \leq \max \left(p^{E D}, 2^{u}\right)$. Pour le $S^{\prime}$-régulateur, nous avons

$$
R_{S^{\prime}} \leq h R \prod_{\mathfrak{p}^{\prime} \in S^{\prime}} \log N_{L / \mathbb{Q}}\left(\mathfrak{p}^{\prime}\right) \leq h R(\log P)^{s}
$$

où la première inégalité vient du lemme 3 de [Bugeaud et Győry 1996]. Pour majorer $h R$, nous employons une estimation de Lenstra [1992, théorème 6.5]. Elle entraîne

$$
h R \leq\left|\Delta_{L / \mathbb{Q}}\right|^{1 / 2}\left(\log ^{\star}\left|\Delta_{L / \mathbb{Q}}\right|\right)^{E D-1} .
$$


La forme faible $h R \leq\left|\Delta_{L / \mathbb{Q}}\right|^{E D / 2}$ permet de majorer

$$
1+\frac{\log ^{\star} R_{S^{\prime}}}{\log ^{\star} P} \leq 1+\log ^{\star}\left(\left|\Delta_{L / \mathbb{Q}}\right|^{E D / 2}\right)+s \frac{\log ^{\star} \log ^{\star} P}{\log ^{\star} P} \leq 2 u \log ^{\star}\left|\Delta_{L / \mathbb{Q}}\right| .
$$

En rassemblant les différents termes, il vient

$$
h(b) \leq 2^{16} u(4 u)^{4 u+8} P(\log P)^{u}\left|\Delta_{L / \mathbb{Q}}\right|^{1 / 2}\left(\log ^{\star}\left|\Delta_{L / \mathbb{Q}}\right|\right)^{E D} .
$$

Nous faisons alors intervenir $P \leq p^{u}, \log P \leq p u$ et si $x \geq 1$

$$
\left(\log ^{\star} x\right)^{E D} \leq(E D)^{E D} x^{1 / 2}
$$

(écrire $\log y \leq \sqrt{y}$ pour $y=x^{1 / E D}$ ). Nous aboutissons à

$$
h(b) \leq 2{ }^{16} u(4 u)^{4 u+8} p^{2 u} u^{2 u}\left|\Delta_{L / \mathbb{Q}}\right|
$$

puis, en tirant parti de $u \geq 4$, à

$$
h(b) \leq(4 u)^{9 u} p^{2 u}\left|\Delta_{L / \mathbb{Q}}\right| .
$$

Pour majorer le discriminant de $L$, nous devons faire intervenir le fait que $L / K$ est non ramifiée en dehors de $S^{\prime}$ (proposition 2.1). Notons pour cela 2 l'ensemble des caractéristiques résiduelles des places de $S^{\prime}$. Un résultat de Serre [1981, proposition $4^{\prime}$, page 129] s'écrit avec les présentes notations :

$$
\log \left|\Delta_{L / \mathbb{Q}}\right| \leq E \log \Delta+D(E-1) \sum_{\ell \in \mathscr{2}} \log \ell+(\operatorname{Card} 2) E D \log E .
$$

Ici Card $2 \leq \sum_{\ell \in \mathscr{2}} \log \ell+1 \leq \Omega+\log p$ et donc

$$
\log \left|\Delta_{L / \mathbb{Q}}\right| \leq E \log \Delta+2 E D(\Omega+\log p) \log E .
$$

Nous majorons ensuite (quelque peu brutalement) $D$ par $\Omega$ dans cette formule, $u$ par $3 E \Omega / 2$ et $\Omega \log \Omega$ par $\Omega^{2}$ pour obtenir

$$
\begin{aligned}
\log h(b) \leq & 14 E \Omega \log 6 E+14 E \Omega^{2}+3 E \Omega \log p \\
& +E \log \Delta+2 E \Omega^{2} \log E+2 E \Omega(\log p)(\log E) \\
\leq & 63 E \Omega^{2}\left(\log ^{\star} p\right)\left(\log ^{\star} E\right)+E \log \Delta \\
\leq & 2^{6} E\left(\log \Delta_{S}\right)\left(\log ^{\star} p\right)\left(\log ^{\star} E\right) .
\end{aligned}
$$

Pour terminer la preuve de la proposition 3.1, nous vérifions (élémentairement)

$$
2^{6} E\left(\log ^{\star} p\right)\left(\log ^{\star} E\right) \leq(8 g)^{5}
$$

à l'aide de $E \leq 6\left(\begin{array}{l}r \\ 4\end{array}\right), r \leq 2 g+2$ et $p \leq 2 g+1$. 


\section{Hauteur de la courbe}

Dans cette dernière partie, nous oublions entièrement le corps $K$ pour ne travailler que sur $\bar{K}$. Aussi utilisons-nous les notations $\pi: C \rightarrow \mathbb{P}^{1}$ pour désigner l'extension des objets précédents. Quitte à composer $\pi$ avec un automorphisme de $\mathbb{P}^{1}$, nous supposons que 0,1 et $\infty$ font partie des $r \geq 3$ points de branchement. Par suite, les $r-3$ autres appartiennent à l'ensemble $\mathscr{B}$. Nous notons aussi $H \geq 1$ la borne que nous avons obtenue pour la hauteur des éléments de $\mathscr{B}$ (voir proposition 3.1).

Le corps des fonctions de $C$ est une extension galoisienne de $\bar{K}(X)$ (corps des fonctions de $\left.\mathbb{P}^{1}\right)$ de groupe $\mathbb{Z} / p \mathbb{Z}$. Comme $\bar{K}(X)$ contient les racines $p$-ièmes de l'unité, il s'agit d'une extension de Kummer et donc il existe une fraction rationnelle non nulle $F \in \bar{K}(X)$ telle que le corps des fonctions de $C$ soit isomorphe à

$$
\bar{K}(X)[Y] /\left(Y^{p}-F(X)\right) .
$$

Bien entendu, nous pouvons modifier dans cette assertion $F$ par une puissance $p$-ième ce qui permet de supposer que $F$ est un polynôme unitaire dont toutes les racines sont de multiplicité au plus $p-1$. Écrivons

$$
F(X)=\prod_{i=1}^{t}\left(X-b_{i}\right)^{a_{i}}
$$

où $b_{i} \in \bar{K}$ et $1 \leq a_{i} \leq p-1$. Notre courbe $C$ est l'unique courbe projective lisse birationnelle à la courbe affine $C_{0}$ d'équation $Y^{p}=F(X)$ et $\pi$ se factorise à travers $C_{0} \rightarrow \mathbb{A}^{1},(X, Y) \mapsto X$. Ceci entraîne immédiatement que les points de branchement de $\pi$ sont contenus dans $\left\{b_{1}, \ldots, b_{t}, \infty\right\}$. Réciproquement chaque $b_{i}$ correspond à un point de branchement car il est l'image d'un unique point de la normalisée de $C_{0}$ (localement pour la topologie étale $C_{0}$ est isomorphe à la courbe $Y^{p}=X^{a_{i}}$ dont la normalisée est $\mathbb{A}^{1}$ ). Par conséquent $b_{i} \in \mathscr{B} \cup\{0,1\}$ et donc $h\left(b_{i}\right) \leq H$. Ceci montre aussi $t=r-1$.

Notons à présent $G$ le polynôme $Y^{p}-F(X)$ de $\bar{K}[X, Y]$. Nous estimons son degré

$$
\operatorname{deg} G \leq \max (p,(r-1)(p-1)) \leq 2(r-2)(p-1)=4 g
$$

et sa hauteur naïve (celle du point projectif formé par ses coefficients, voir [Rémond 2010])

$$
h_{\infty}(G)=h_{\infty}(F) \leq(\operatorname{deg} F) \log 2+\sum_{i=1}^{r-1} a_{i} h\left(b_{i}\right) \leq 7 g H .
$$

Par conséquent, le théorème 1.5 de [ibid.] affirme qu'il existe un plongement de $C$ dans $\mathbb{P}_{\bar{K}}^{3}$ de degré au plus $8 g(2 g-1)$ et de hauteur (au sens de la hauteur 
projective d'un fermé de $\mathbb{P}^{3}$ )

$$
h(C) \leq(4 g)^{(4 g)^{3}-5}\left(42 g H+9(4 g)^{2}\right) \leq(4 g)^{(4 g)^{3}} H-1 .
$$

Nous pouvons ensuite passer à la hauteur thêta de la jacobienne de $C$ grâce au théorème 1.3 de [Rémond 2010]. L'entier $m$ y apparaissant est majoré par

$$
4 g-2+16 g(2 g-1) \leq 32 g^{2}
$$

de sorte que, en écrivant $h_{\theta}=h_{\theta}^{(4)}\left(\mathrm{Jac} C, \Theta_{\mathrm{sym}}\right)$ la hauteur thêta associée au plongement thêta donné par le diviseur $16 \Theta_{\text {sym }}$ (voir encore [ibid.]), nous avons

$$
h_{\theta} \leq\left(32 g^{2}\right)^{640 g^{2} g^{g}+32 g^{3}} H \leq 2^{3360 \cdot g^{3} 8^{g}} H
$$

(en utilisant $32 g^{2} \leq 2^{5 g}$ ). Le résultat de [Pazuki 2012] compare $h_{\theta}$ (correspondant à $r=4$ dans cet article) et la hauteur de Faltings stable :

$$
h_{\text {Falt }}(C) \leq 2 h_{\theta}+2 C_{1} \log \left(2+\max \left(1, h_{\theta}\right)\right)
$$

pour une constante $C_{1}$ dont on vérifie facilement qu'elle satisfait $2^{4 g} \leq C_{1} \leq 2^{10 g}$. En particulier, si $h_{\theta} \leq \mathscr{H}$ et $\mathscr{H} \geq C_{1}^{2}$ alors $h_{\text {Falt }}(C) \leq 4 \mathscr{H}$. Ceci est très largement vérifié pour $\mathscr{H}=2^{3360 \cdot g^{3} 8^{g}} H$. On en déduit $h_{\text {Falt }}(C) \leq 2^{3362 \cdot g^{3} 8^{g}} H$. Une élémentaire étude de fonction donne $g^{3} 8^{g} \leq 823 \cdot 9^{g}$ et, comme $3362 \cdot 823 \leq 2^{22}$, cela termine la démonstration du théorème 1.2 .

Remarque. Nous avons établi une majoration de la forme $h_{\text {Falt }}(C) \leq c(g) H$ pour une valeur explicite de $c(g)$. Cette dernière ne prétend aucunement être optimale et peut très certainement être améliorée. Une méthode pourrait être de raffiner la preuve de [Rémond 2010] dans le cas d'un polynôme particulier comme $G$. Une autre approche consisterait à travailler plus directement avec la hauteur de Faltings, par exemple en essayant d'expliciter une base des formes différentielles globales sur notre courbe.

\section{Remerciements}

Nous remercions chaleureusement Bjorn Poonen pour ses commentaires sur une première version de ce texte.

\section{Bibliographie}

[Bugeaud et Győry 1996] Y. Bugeaud et K. Győry, "Bounds for the solutions of unit equations", Acta Arith. 74 :1 (1996), 67-80. MR 97b :11045 Zbl 0861.11023

[Faltings 1983] G. Faltings, "Endlichkeitssätze für abelsche Varietäten über Zahlkörpern", Invent. Math. 73 :3 (1983), 349-366. MR 85g :11026a Zbl 0588.14026

[González Díez 1991] G. González Díez, "Loci of curves which are prime Galois coverings of $\mathbf{P}^{1}$ ", Proc. London Math. Soc. (3) 62 :3 (1991), 469-489. MR 92c :14021 Zbl 0679.14010 
[Győry et Yu 2006] K. Győry et K. Yu, "Bounds for the solutions of $S$-unit equations and decomposable form equations”, Acta Arith. 123 :1 (2006), 9-41. MR 2007d :11032 Zbl 1163.11026

[Lang 1983] S. Lang, Complex multiplication, Grundlehren Math. Wiss. 255, Springer, New York, 1983. MR 85f :11042 Zbl 0536.14029

[Lenstra 1992] H. W. Lenstra, Jr., "Algorithms in algebraic number theory", Bull. Amer. Math. Soc. (N.S.) 26 :2 (1992), 211-244. MR 93g :11131 Zbl 0759.11046

[Lønsted et Kleiman 1979] K. Lønsted et S. L. Kleiman, "Basics on families of hyperelliptic curves", Compositio Math. 38 :1 (1979), 83-111. MR 80g :14028 Zbl 0406.14017

[Oort 1974] F. Oort, "Hyperelliptic curves over number fields", pp. 211-218 dans Classification of algebraic varieties and compact complex manifolds, édité par H. Popp, Lecture Notes in Math. 412, Springer, Berlin, 1974. MR 50 \#7154 Zbl 0299.14017

[Pazuki 2012] F. Pazuki, “Theta height and Faltings height”, Bull. Soc. Math. France 140 :1 (2012), 19-49. Zbl 06032258

[Rémond 1999] G. Rémond, "Hauteurs thêta et construction de Kodaira", J. Number Theory 78 :2 (1999), 287-311. MR 2000g :11059 Zbl 0947.14016

[Rémond 2010] G. Rémond, "Nombre de points rationnels des courbes", Proc. Lond. Math. Soc. (3) 101 :3 (2010), 759-794. MR 2011k :11088 Zbl 1210.11073

[Serre 1981] J.-P. Serre, "Quelques applications du théorème de densité de Chebotarev", Inst. Hautes Études Sci. Publ. Math. 54 (1981), 323-401. MR 83k :12011 Zbl 0496.12011

[Shafarevitch 1963] I. R. Shafarevitch, "Поля алгебраических чисел", pp. 163-176 dans Proc. Internat. Congr. Mathematicians (Stockholm, 1962), Inst. Mittag-Leffler, Djursholm, 1963. Traduction anglaise : "Algebraic number fields", dans Amer. Math. Soc. Transl. 231 (1963), 25-39. MR 34 \#2569 Zbl 0126.06902

Communicated by Hendrik W. Lenstra

Received 2011-01-06 Revised 2011-03-07 Accepted 2011-03-07

rdejong@math.leidenuniv.nl Mathematisch Instituut, Universiteit Leiden, PO Box 9512, 2300 RA Leiden, Netherlands

Gael.Remond@ujf-grenoble.fr Institut Fourier, UMR 5582, Université Grenoble I, BP 74, 38402 Saint-Martin-d'Hères Cedex, France 


\section{Algebra \& Number Theory}

msp.berkeley.edu/ant

\section{EDITORS}

MANAGING EDITOR

Bjorn Poonen

Massachusetts Institute of Technology

Cambridge, USA

\author{
EDITORIAL BOARD CHAIR \\ David Eisenbud \\ University of California \\ Berkeley, USA
}

\section{BOARD OF EDITORS}

Georgia Benkart

Dave Benson

Richard E. Borcherds

John H. Coates

J-L. Colliot-Thélène

Brian D. Conrad

Hélène Esnault

Hubert Flenner

Edward Frenkel

Andrew Granville

Joseph Gubeladze

Ehud Hrushovski

Craig Huneke

Mikhail Kapranov

Yujiro Kawamata

János Kollár

Yuri Manin

Barry Mazur

Philippe Michel

Susan Montgomery
University of Wisconsin, Madison, USA

University of Aberdeen, Scotland

University of California, Berkeley, USA

University of Cambridge, UK

CNRS, Université Paris-Sud, France

University of Michigan, USA

Universität Duisburg-Essen, Germany

Ruhr-Universität, Germany

University of California, Berkeley, USA

Université de Montréal, Canada

San Francisco State University, USA

Hebrew University, Israel

University of Kansas, USA

Yale University, USA

University of Tokyo, Japan

Princeton University, USA

Northwestern University, USA

Harvard University, USA

École Polytechnique Fédérale de Lausanne

University of Southern California, USA
Shigefumi Mori

Raman Parimala

Jonathan Pila

Victor Reiner

Karl Rubin

Peter Sarnak

Joseph H. Silverman

Michael Singer

Ronald Solomon

Vasudevan Srinivas

J. Toby Stafford

Bernd Sturmfels

Richard Taylor

Ravi Vakil

Michel van den Bergh

Marie-France Vignéras

Kei-Ichi Watanabe

Andrei Zelevinsky

Efim Zelmanov
RIMS, Kyoto University, Japan

Emory University, USA

University of Oxford, UK

University of Minnesota, USA

University of California, Irvine, USA

Princeton University, USA

Brown University, USA

North Carolina State University, USA

Ohio State University, USA

Tata Inst. of Fund. Research, India

University of Michigan, USA

University of California, Berkeley, USA

Harvard University, USA

Stanford University, USA

Hasselt University, Belgium

Université Paris VII, France

Nihon University, Japan

Northeastern University, USA

University of California, San Diego, USA

\section{PRODUCTION}

contact@msp.org

Silvio Levy, Scientific Editor

See inside back cover or www.jant.org for submission instructions.

The subscription price for 2011 is US \$150/year for the electronic version, and \$210/year (+\$35 shipping outside the US) for print and electronic. Subscriptions, requests for back issues from the last three years and changes of subscribers address should be sent to Mathematical Sciences Publishers, Department of Mathematics, University of California, Berkeley, CA 94720-3840, USA.

Algebra \& Number Theory (ISSN 1937-0652) at Mathematical Sciences Publishers, Department of Mathematics, University of California, Berkeley, CA 94720-3840 is published continuously online. Periodical rate postage paid at Berkeley, CA 94704, and additional mailing offices.

ANT peer review and production are managed by EditFLOW ${ }^{\circledR}$ from Mathematical Sciences Publishers.

PUBLISHED BY

mathematical sciences publishers

http://msp.org/

A NON-PROFIT CORPORATION

Typeset in IAT $_{\mathrm{E}} \mathrm{X}$

Copyright @2011 by Mathematical Sciences Publishers 


\section{Algebra \& Number Theory}

\section{Volume $5 \quad$ No. $8 \quad 2011$}

The behavior of Hecke $L$-functions of real quadratic fields at $s=0$ BYUNGHEUP JUN and JUNGYUN LEE

The Picard group of a $K 3$ surface and its reduction modulo $p$

ANDREAS-STEPHAN ELSENHANS and JÖRG JAHNEL

Linear determinantal equations for all projective schemes JESSICA SIDMAN and GREGORY G. SMITH

Involutions, weights and $p$-local structure

GEOFFREY R. ROBINSON

Parametrizing quartic algebras over an arbitrary base

MELANIE MatchetT WoOD

Coleman maps and the $p$-adic regulator

ANTONIO LEI, DAVID LOEFFLER and SARAH LIVIA ZERBES

Conjecture de Shafarevitch effective pour les revêtements cycliques 